\title{
Current challenges in peptide-based drug discovery
}

\author{
Laszlo Otvos Jr' and John D. Wade ${ }^{2 *}$ \\ ${ }^{1}$ Department of Biology, Temple University, Philadelphia, PA, USA \\ ${ }^{2}$ Florey Institute of Neuroscience and Mental Health, The University of Melbourne, Melbourne, VIC, Australia \\ *Correspondence: john.wade@florey.edu.au
}

Edited and reviewed by:

Charlotte A. E. Hauser, Institute of Bioengineering and Nanotechnology, Singapore

Keywords: peptide drug development, oral delivery, pharmacokinetics, pharmacodynamics, peptides, grand challenges

The central event of each signaling step in biology is biomolecular recognition. Notwithstanding the importance of nucleic acids, carbohydrates, or lipids in ligand-target interactions, the effectors of most signal transduction processes are peptides. These can be fragments of proteins or stand-alone hormones, cytokines, toxins, antimicrobials, and many other types of peptides. At this point there is no good reason to classify peptides by the number of amino acid residues. We consider peptides as any polyamide (or even biopolymer with ester, thioester, or otherwise modified backbone) that can be made on a contemporary chemical peptide synthesizer. The limit in size is greater than the arbitrary cutoff of 50 amino acids set up by the US Food and Drug Administration (Carton and Strohl, 2013) for proteins and far exceeds that of biological recognition elements. While target recognition can occur with as low as a few residues (Ertl et al., 1991), even wide binding groves can be bound by 30-40 residue long peptides. Thus, in principle synthetic peptides can be used to regulate almost all receptor responses.

The high specificity and low toxicity of peptide drugs derive from their extremely tight binding to their targets. This is due to the large chemical space the side-chain variations of native amino acids cover. Current databases estimate the total number of valid protein-ligand binding sites at 7700 (Khazanov and Carlson, 2013). Calculation based on 17 variable residues (Cys, Met, and Trp are significantly underrepresented in known ligands), show that an 83,000-member tetrapeptide library can be prepared that will essentially cover all unique protein binding regions. As the median length of an active site is 11 amino-acid residues (Khazanov and Carlson, 2013), designed ligands should also be longer. While historically six-residue positional scanning could identify ligands of receptors or epitopes of monoclonal antibodies (Dooley and Houghten, 1993), in our experience receptor agonists are 9-12 residue long (Otvos et al., 2008, 2011a) much like major histocompatibility complex binding peptides (Appella et al., 1995). Antagonists acting on the same receptor binding sites are somewhat shorter (vide infra). If it is assumed that conformational preferences improve the binding kinetics but only rarely thermodynamics, then the tremendous specificity of side-chain combinations of peptides over six residues in length can be even further expanded by using non-natural residues. Hundreds of appropriately protected and activated non-natural amino acid derivatives, ready for incorporation into synthetic peptides, are commercially available and indeed are frequently explored in peptidebased drug design. Importantly, chemical biology has provided both backbone and side-chain combinations for exploring an enormous chemical space and is expected to supply peptide chemists with further building blocks suitable for identifying close-to-ideal agonists and antagonists of any biologically important target.

The selectivity of peptide drugs for their target is highlighted by the elevated success rate in clinical trials. According to a biotechnology report (Thomas, 2013), of the 40 approved drugs in 2012, five (12.5\%) were peptides compared to 28 small molecule drugs and two monoclonal antibodies (in addition to three enzymes, a cell-based drug and a vaccine).
However, in a recent report, the total number of peptide approvals between 2001 and 2012 was 19 (Kaspar and Reichert, 2013). Due to the low number of drug approvals, any particularly successful year can bias the ratios significantly. According to another report, the overall success rate of all drugs entering clinical trials is just $10.4 \%$ (Hay et al., 2014). Sixty-five percent of small molecules proceed from Phase I to Phase II in non-oncology applications, a figure identical for peptide/protein drugs. Interestingly peptides/proteins outperform small molecules at the Phase II $\rightarrow$ Phase III transition stage with $29 \%$ for small molecules and $42 \%$ for the larger drug candidates. While peptides have traditionally been considered safe in Phase I clinical trials, the public perception is that they are less beneficial in late clinical trials when they are compared side-by-side with different types of treatment modalities. It must be mentioned that peptides are less successful in oncology than in other applications. The cost of large scale peptide production might well-exceed those of small molecule drugs, but if one considers the total cost of the drug development process, the active pharmaceutical ingredient expense will remain under 3\% (Otvos, 2014a). In direct opposition to concerns with expensive peptides, the increased clinical success rate, and thus, overall expense/approved drug ratio compared to small molecule chemical entities, make peptide drug development particularly attractive.

The biochemical processes that activated receptors directly or indirectly regulate include protein phosphorylation, nucleic acid transcription, ion transport, and a series of enzyme activities (Yan 
and Wang, 2012). Ligands (e.g., drugs, hormones, neurotransmitters) bind to receptors and ligand binding activates or inactivates the target and accelerates or inhibits given cellular functions. For peptide-based drug development, a compromise has to be found between the required peptide length and pharmacologically useful levels of receptor activation. The numerous variables include (i) the size and accessibility of ligand binding surfaces, (ii) possible induced fit; (iii) ligand stability and receptor residency time. Contemporary drug development requires nano- or picomolar cellular responses especially for receptor-mediated biological processes. As an example, our leptin receptor agonist peptides must be at least 11 amino acid residues in length (Otvos et al., 2008), similar to other peptide hormone receptor activators (Demchyshyn et al., 2000; Shimizu et al., 2001). For turning peptide agonists into antagonists, the literature data (Hruby, 2002; Sillerud and Larson, 2005) support our own personal experience. Truncation of the sequence or non-natural amino acid replacement usually leads to antagonist or inverse agonist derivatives. Our nine-residue leptin receptor antagonist Allo-aca follows these design rules and shows opposite effects to the agonist both in vitro and in vivo (Otvos et al., 2011b). Moreover, a library based on the agonist or conformational restriction may allow the selection of peptides with antagonist properties.

In spite of all listed attractive features, ongoing negative public opinion limits more widespread acceptance of peptide drugs. A major challenge in the coming decade will be to modify peptide (polyamide) sequences or properties to overcome these valid concerns and to educate the public to dismiss or reduce the unfounded misconceptions. One of the true drawbacks of peptide drugs is the increased proteolytic instability compared to not only small molecules but also monoclonal antibody therapeutics. The Fc fragment of monoclonal antibodies reshuffle the protein to cells and peptideFc conjugates may also be protected from enzymatic cleavage (Boylan et al., 2013). Chemically, both the amide bond and the side-chains can be altered to render the resulting peptidomimetics resistant to proteolytic degradation (Gentilucci et al., 2010). The serum stability assay provides a ready measure of peptide stability and was once considered the most significant secondary screening tool in drug development (Powell et al., 1992). Indeed, serum stability can provide a strong prediction of the all-important pharmacokinetic behavior of drugs.

Nevertheless, the discord between the pharmacokinetics (what the body does to the drug) and the pharmacodynamics (what the drug does to the body) of peptide therapeutics warrants revisiting the importance of stability per se. In a mouse model of triple negative breast cancer, Allo-aca is more efficacious than any other current therapy regimen as indicated by survival figures (Otvos et al., 2011c). Due to the similarity of molecular mechanisms in arthritis and cancer, Allo-aca softens rheumatoid arthritis development markers in mice indicating clear and longterm activity of the peptide in vivo (Otvos et al., 2011d). At the same time, Alloaca decomposes within $30 \mathrm{~min}$ in human serum and is undetectable in pharmacokinetic measurements $1 \mathrm{~h}$ after subcutaneous administration (Otvos et al., 2014). The remarkable in vivo efficacies can be explained by the dynamics of Allo-aca binding to the leptin receptor. The estimated binding affinity of biotin-labeled Allo-aca to the ligand binding domain of the receptor is $300 \mathrm{pM}$ and the dissociation rate constant of $1.5 \times 10-4 / \mathrm{s}$ corresponds to a peptide-receptor complex half-life of nearly $2 \mathrm{~h}$. Allo-aca, and other peptide drugs excel in terms of high activity and target selectivity regardless of poor serum stability and pharmacokinetics. Peptide-based drugs may modify receptor responses significantly longer than standard stability analyses indicate. Indeed, Allo-aca produces weight gain in normal mice even two days after a $0.1 \mathrm{mg} / \mathrm{kg}$ bolus subcutaneous administration despite the very short-lived blood levels. In addition, because peptides are rapidly excreted through the kidney, serum stability studies are not representative of true turnover. The 5-10 min $\mathrm{T}_{\max }$ of peptide drug leads in mouse pharmacokinetics measurements is significantly shorter than true serum presence in humans where the renal clearance rate is 10-fold longer (Sakamoto et al., 1993). Even if they are cleaved into smaller fragments, peptidic metabolites frequently retain the intended biological function (Noto et al., 2008).

Improvement in peptide drug penetration through biological barriers can be achieved by adding modules for passive or active transport (Fasano, 1998). Incorporation of positively charged amino acids, especially at terminal positions improves cell and tissue penetration of peptides (Teesalu et al., 2009; Li and Cho, 2012). Repetitive arginine-containing modules help even nuclear uptake in vitro or bioavailability in vivo (Wender et al., 2000). One problem is that polycations frequently destroy mammalian membranes as shown by the toxic properties of natural or designer antibacterial peptides that contain large numbers of lysines and arginines (Cudic et al., 2002). Presumably, a safer solution is to conjugate therapeutic peptides to ligands of cell surface receptors. While absorptive-mediated uptake features micromolar saturation constants, generally receptor-mediated uptake is characterized by $\mathrm{K}_{\mathrm{d}}$ values in the low nanomolar range. Cell surface receptors that can be targeted for internalization of peptides include carbohydrate receptors, lipoprotein receptors, transferring receptors, and receptors involved in cell adhesion. Perhaps the most widely used of these is the incorporation of various sugars to improve tissue penetration, including the transport across the blood-brain barrier. In the first example, coupling a maltose moiety to the $\mathrm{N}$-terminus of a somatostatin octapeptide analog resulted in about 10-fold increased oral bioavailability while maintaining the selectivity and duration of action of the original peptide (Albert et al., 1993). Later this technology was extended to increase in intraintestinal absorption of vasopressin (Kihlberg et al., 1995), the stability of peptide drugs and brain transport of enkephalin analogs (Egleton and Davis, 2005), and more recently to simultaneous intestinal drug absorption and blood-brain barrier penetration of endomorphine-1, an opioid tetrapeptide (Varamini et al., 2012). In our experience, a leptin receptor agonist glycopeptide E1/Aca reduces weight gain in mice fed with high-fat peanut diet in a dose-dependent manner, unlike native leptin protein. In mice undergoing 
leptin glycopeptide treatment, several obesity-related pathologies (i.e., abnormal metabolic profile and liver histology as well as infertility) are normalized while unglycosylated leptin protein therapy does not show similar positive treatment outcomes (Kovalszky et al., 2010).

Another related area where chemical biology can make critical contributions is increasing the sensitivity of quantitative analysis of peptides. The current limit of quantitation of peptides in plasma by using nano-high-performance liquid chromatography assays is approximately $25 \mathrm{nM}$ (Otvos et al., 2014), around the lower limit of the $10 \mathrm{ng} / \mathrm{mL}$ dynamic range (in a 10-mer peptide this is equivalent of $10 \mathrm{nM})$ in validated pharmaceutical protocols (Zannikos et al., 2000). However, receptor agonist and antagonist peptide drugs act in the pM range, and they are present in mouse blood at a concentration higher than the $100-500 \mathrm{pM} \mathrm{IC}_{50}$ value well-beyond the 30-60 min mark that corresponds to the limit of quantitation of the analysis. The situation is less problematic in humans where higher blood volumes are available than in rodents, although the required drug doses in humans are about 12-fold lower than in mice due to differences in the body surface area/weight ratio (Reagan-Shaw et al., 2008). Human serum concentrations of triptorelin, a 10-residue agonist of the gonadotropinreleasing hormone receptor, at $8 \mathrm{ng} / \mathrm{mL}$ are already associated with activation of $90 \%$ of the receptor population (Romero et al., 2012). In turn, even if the sensitivity of the murine plasma quantification protocol can be increased by a magnitude, later time points will still be missed when highly active peptide drugs are present in the circulation above their $\mathrm{IC}_{50} / \mathrm{EC}_{50}$ figures.

Finally, oral bioavailability (in fact the lack thereof) is a constant discussion topic between peptide drug developers and pharma. Peptides can rarely be absorbed by the intestinal mucosa, and thus cannot serve the appealing lifestyle drug market that includes treatments for weight-loss, smoking, erectile dysfunction, wrinkles, and baldness (Atkinson, 2002). Much effort and monetary resources are expended in the endeavor to make peptides orally active. Chemical and physical modifications that can improve oral bioavailability of peptide drugs include conjugation to passive and active transport enhancers (vide supra). In addition to these covalent modification technologies, micro- and nanoparticles further increase peptide delivery options. In most cases, as soon as a peptide drug lead is identified, research is initiated to improve its oral availability. In the latest example, mucoadhesive devices, made of carbopol, pectin and sodium methylcarboxy cellulose, in enteric coated capsules significantly improve the oral bioavailability and pharmacodynamics parameters of an existing peptide drug, salmon calcitonin (Gupta et al., 2013).

However, it must be said that peptide drugs do not necessarily need to be orally available. Many peptide hormones, including insulin, amylin, somatostatin, and human growth hormone are now available in patient-friendly packaging ready for subcutaneous self-administration. The luteinizing hormone-releasing hormone receptor agonist leuprolide, is currently available in a once-a-year implantable device for commercial use. At a research scale, the transdermal delivery of leuprolide can be further improved by using microneedles and/or iontophoresis (Sachdeva et al., 2013). Many peptides can be formulated for intranasal administration, a technique that can utilize olfactory neurons to bypass blood-brain barrier restrictions for central nervous system therapeutics (Charlton et al., 2008). Unsurpassed intranasal efficacies for peptides, comparable to those measured after injection, are also achieved by using alkylsaccharide transmucosal delivery agents (Maggio, 2006). In fact the market is saturated now with personalized, reusable, and virtually painless transdermal or intranasal medical devices for drug administration.

Taken together, the therapeutic potential of peptide-based drugs is increasingly appreciated and their development is both strong and growing rapidly. At this juncture, the role of chemical biology is twofold: solve or improve some of the suboptimal parameters of peptides such as poor pharmacokinetics or lack of oral activity simultaneously with educating biotechnology investors and less informed commercial drug developers about the misconceptions (immunogenicity, high price, unfavorable pharmacodynamics, lack of delivery options) that still linger over peptide-based therapeutics and delay the realization of the use of these highly active and safe therapy options (Otvos, 2014b). By addressing these outstanding issues, peptide-based drugs will finally be accepted as genuine alternatives to traditional small molecule therapeutics.

\section{REFERENCES}

Albert, R., Marbach, P., Bauer, W., Briner, U., Fricker, G., Bruns, C., et al. (1993). SDZ CO 611: a highly potent glycated analog of somatostatin with improved oral activity. Life Sci. 53, 517-525. doi: 10.1016/0024-3205(93)90703-6

Appella, E., Padlan, E. A., and Hunt, D. F. (1995). Analysis of the structure of naturally processed peptides bound by class I and class II major histocompatibility complex molecules. Experimentia 73, 105-119.

Atkinson, T. (2002). Lifestyle drug market booming. Nat. Med. 8, 909. doi: 10.1038/nm0902-909

Boylan, N. J., Zhou, W., Proos, R. J., Tolbert, T. J., Wolfe, J. L., and Laurence, J. S. (2013). Conjugation site heterogeneity causes variable electrostatic properties in Fc conjugates. Bioconjug. Chem. 24, 1008-1016. doi: 10.1021/bc4000564

Carton, J. M., and Strohl, W. R. (2013). "Protein therapeutics (Introduction to biopharmaceuticals)," in Biological and Small Molecule Drug Research and Development, eds R. Ganelin, R. Jefferts, and S. Roberts (Waltham, MA: Academic Press), 127-159.

Charlton, S. T., Whetstone, J., Fayinka, S. T., Read, K. D., Illum, L., and Davis, S. S. (2008). Evaluation of direct transport pathways of glycine receptor antagonists and an angiotensin antagonist from the nasal cavity to the central nervous system in the rat model. Pharm. Res. 25, 1531-1543. doi: 10.1007/s11095-008-9550-2

Cudic, M., Condie, B. A., Weiner, D. J., Lysenko, E. S., Xiang, Z. Q., Insug, O., et al. (2002) Development of novel antibacterial peptides that kill resistant isolates. Peptides 23, 2071-2083. doi: 10.1016/S0196-9781(02)00244-9

Demchyshyn, L. L., McConkey, F., and Niznik, H. B. (2000). Dopamine D5 receptor agonist high affinity and constitutive activity profile conferred by carboxy-terminal tail sequence. J. Biol. Chem. 275, 23446-23455. doi: 10.1074/jbc.M000157200

Dooley, C. T., and Houghten, R. A. (1993). The use of positional scanning synthetic peptide combinatorial libraries for the rapid determination of opioid receptor ligands. Life Sci. 52, 1509-1517. doi: 10.1016/0024-3205(93)90113-H

Egleton, R. D., and Davis, T. P. (2005). Development of neuropeptide drugs that cross the blood-brain barrier. NeuroRx. 2, 44-53. doi: 10.1602/neurorx.2.1.44

Ertl, H. C., Dietzschold, B., and Otvos, L. Jr. (1991). $\mathrm{T}$ helper cell epitope of rabies virus nucleoprotein defined by tri- and tetrapeptides. Eur. J. Immunol. 21, 1-10. doi: 10.1002/eji.1830210102

Fasano, A. (1998). Innovative strategies for the oral delivery of drugs and peptides. Trends Biotechnol. 16, 152-157. doi: 10.1016/S0167-7799(97)01170-0 
Gentilucci, L., De Marco, R., and Cerisoli, L. (2010). Chemical modifications designed to improve peptide stability: incorporation of non-natural amino acids, pseudo-peptide bonds, and cyclization. Curr. Pharm. Des. 16, 3185-3203. doi: 10.2174/138 161210793292555

Gupta, V., Hwang, B. H., Lee, J., Anselmo, A. C., Doshi, N., and Mitragotri, S. (2013). Mucoadhesive intestinal devices for oral delivery of salmon calcitonin. J. Control. Release. 172, 753-762. doi: 10.1016/j.jconrel.2013.09.004

Hay, M., Thomas, D. W., Craighead, L., Economides, C., and Rosenthal, J. (2014). Clinical development success rates for investigational drugs. Nat. Biotechnol. 32, 40-51. doi: 10.1038/ nbt.2786

Hruby, V. J. (2002). Designing peptide receptor agonists and antagonists. Nat. Rev. Drug Discov. 1, 847-858. doi: 10.1038/nrd939

Kaspar, A. A., and Reichert, J. M. (2013). Future directions for peptide therapeutics development. Drug Discov. Today 18, 807-817. doi: 10.1016/j.drudis. 2013.05.011

Khazanov, N. A., and Carlson, H. A. (2013). Exploring the composition of protein-ligand binding sites on a large scale. PLoS Comput. Biol. 9:e1003321. doi: 10.1371/journal.pcbi.1003321

Kihlberg, J., Ahman, J., Walse, B., Drakenberg, T., Nilsson, A., Söderberg-Ahlm, C., et al. (1995). Glycosylated peptide hormones: pharmacological properties and conformational studies of analogues of (1-desamino, 8-D-arginine) vasopressin. J. Med. Chem. 38, 161-169. doi: 10.1021/jm0000 $1 \mathrm{a} 021$

Kovalszky, I., Surmacz, E., Scolaro, L., Cassone, M., Sztodola, A., Olah, J., et al. (2010). Leptin-based glycopeptide induces weight loss and simultaneously restores fertility in animal models. Diabetes Obes. Metab. 12, 393-402. doi: 10.1111/j.14631326.2009.01170.x

Li, Z. J., and Cho, C. H. (2012). Peptides as targeting probes against tumor vasculature for diagnosis and drug delivery. J. Transl. Med. 10, 51. doi: 10.1186/1479-5876-10-S1-S1

Maggio, E. T. (2006). Intravail: highly effective intranasal delivery of peptide and protein drugs. Expert Opin. Drug Deliv. 3, 529-539. doi: 10.1517/ 17425247.3.4.529

Noto, P. B., Abbadessa, G., Cassone, M., Mateo, G. D., Agelan, A., Wade, J. D., et al. (2008). Alternative stabilities of a proline-rich antibacterial peptide in vitro and in vivo. Protein Sci. 17, 1249-1255. doi: 10.1110/ps.034330.108

Otvos, L. Jr. (2014a). Peptide-based drug research and development: relative costs, comparative value. Pharm. Outsourc. 15, 16-20. Available online at: http://www.pharmoutsourcing.com/ Featured-Articles/161800-Peptide-based-Drug-Res earch-and-Development-Relative-Costs-Compara tive-Value/

Otvos, L. Jr. (2014b). Peptide-based drug discovery and development. Common misconceptions. Pharm. Outsourc. 15, 40-43. Available online at: http://www.pharmoutsourcing.com/
Featured-Articles/157959-Peptide-based-Drug-Dis covery-Development/

Otvos, L. Jr., Haspinger, E., La Russa, F., Maspero, F., Graziano, P., Kovalszky, I., et al. (2011a). Design and development of a peptide-based adiponectin receptor agonist for cancer treatment. BMC Biotechnol. 11:90. doi: 10.1186/1472-675011-90

Otvos, L. Jr., Kovalszky, I., Riolfi, M., Ferla, R., Olah, J., Sztodola, A., et al. (2011c). Efficacy of a leptin receptor antagonist peptide in a mouse model of triple-negative breast cancer. Eur. J. Cancer 47, 1578-1584. doi: 10.1016/j.ejca.2011.01.018

Otvos, L. Jr., Kovalszky, I., Scolaro, L., Sztodola, A., Olah, J., Cassone, M., et al. (2011b). Peptidebased receptor antagonists for cancer treatment and appetite regulation. Biopolymers 96, 117-125. doi: 10.1002/bip.21377

Otvos, L. Jr., Shao, W.-H., Vanniasinghe, A., Amon, M. A., Holub, M. C., Kovalszky, I., et al. (2011d). Towards understanding the role of leptin and leptin receptor antagonism in preclinical models of rheumatoid arthritis. Peptides 32, 1567-1574. doi: 10.1016/j.peptides.2011.06.015

Otvos, L. Jr., Terrasi, M., Cascio, S., Cassone, M., Abbadessa, G., Di Pascali, F., et al. (2008). Development of a pharmacologically improved peptide agonist of the leptin receptor. Biochim. Biophys. Acta 1783, 1745-1754. doi: 10.1016/j. bbamcr.2008.05.007

Otvos, L. Jr., Vetter, S. W., Koladia, M., Knappe, D., Schmidt, R., Ostorhazi, E., et al. (2014). The designer leptin antagonist peptide Allo-aca compensates for short serum half-life with very tight binding to the receptor. Amino Acids 46, 873-882. doi: 10.1007/s00726-013-1650-6

Powell, M. F., Grey, H., Gaeta, F., Sette, A., and Colon, S. M. (1992). Peptide stability in drug development: a comparison of peptide reactivity in different biological media. J. Pharm. Sci. 81, 731-735. doi: 10.1002/jps.2600810802

Reagan-Shaw, S., Nihal, M., and Ahmad, N. (2008). Dose translation from animal to human studies revisited. FASEB J. 22, 569-661. doi: 10.1096/fi.079574LSF

Romero, E., Velez de Mendizabal, N., Cendros, J. M., Peraire, C., Bascompta, E., Obach, R., et al. (2012). Pharmacokinetic/pharmacodynamic model of the testosterone effects of triptorelin administered in sustained release formulations in patients with prostate cancer. J. Pharmacol. Exp. Ther. 342, 788-798. doi: 10.1124/jpet.112.195560

Sachdeva, V., Zhou, Y., and Banga, A. K. (2013). In vivo transdermal delivery of leuprolide using microneedles and iontophoresis. Curr. Pharm. Biotechnol. 14, 180-193. doi: 10.2174/13892010 11314020008

Sakamoto, H., Hatano, K., Higashi, Y., Mine, Y., Nakamoto, S., Tawara, S., et al. (1993). Animal pharmacokinetics of FK037, a novel parenteral broad-spectrum cephalosporin. J. Antibiot. 46, 120-130. doi: 10.7164/antibiotics.46.120

Shimizu, M., Carter, P. H., Khatri, A., Potts, J. T. Jr., and Gardella, T. J. (2001). Enhanced activity in parathyroid hormone-(1-14) and -(111): novel peptides for probing ligand-receptor interactions. Endocrinology 142, 3068-3074. doi: 10.1210/en.142.7.3068

Sillerud, L. O., and Larson, R. S. (2005). Design and structure of peptide and peptidomimetic antagonists of protein-protein interaction. Curr. Protein Pept. Sci. 6, 151-169. doi: 10.2174/138920305 3545462

Teesalu, T., Sugahara, K. N., Kotamraju, V. R., and Ruoslahti,. E. (2009). C-end rule peptides mediate neuropilin-1-dependent cell, vascular, and tissue penetration. Proc. Natl. Acad. Sci. U.S.A. 106, 16157-16162. doi: 10.1073/pnas.0908201106

Thomas, D. (2013). A big year for novel drug approvals. BIOtechNOW. Available online at: http://www.biotech-now.org/business-and-invest ments/inside-bio-ia/2013/01/a-big-year-for-noveldrugs-approvals\#

Varamini, P., Mansfeld, F. M., Blanchfield, J. T., Wyse, B. D., Smith, M. T., and Toth, I. (2012). Synthesis and biological evaluation of an orally active glycosylated endomorphin-1. J. Med. Chem. 55, 5859-5867. doi: $10.1021 / \mathrm{jm} 300418 \mathrm{~d}$

Wender, P. A., Mitchell, D. J., Pattabiraman, K., Pelkey, E. T., Steinman, L., and Rothbard, J. B. (2000). The design, synthesis, and evaluation of molecules that enable or enhance cellular uptake: peptoid molecular transporters. Proc. Natl. Acad. Sci. U.S.A. 97, 13003-13008. doi: 10.1073/pnas.97.24. 13003

Yan, Z., and Wang, J. (2012). Specificity quantification of biomolecular recognition and its implication for drug discovery. Sci. Rep. 2:309. doi: 10.1038/srep00309

Zannikos, P. N., Rohatagi, S., Jensen, B. K., DePhillips, S. L., and Rhodes, G. R. (2000). Pharmacokinetics and concentration-effect analysis of intravenous RGD891, a platelet GPIIb/IIIa antagonist, using mixed-effects modeling (NONMEM). J. Clin. Pharmacol. 40, 1129-1140. doi: 10.1177/009127000004001007

Conflict of Interest Statement: The authors declare that the research was conducted in the absence of any commercial or financial relationships that could be construed as a potential conflict of interest.

Received: 26 June 2014; accepted: 22 July 2014; published online: 08 August 2014.

Citation: Otvos L Jr and Wade JD (2014) Current challenges in peptide-based drug discovery. Front. Chem. 2:62. doi: $10.3389 /$ fchem.2014.00062

This article was submitted to Chemical Biology, a section of the journal Frontiers in Chemistry.

Copyright (c) 2014 Otvos and Wade. This is an openaccess article distributed under the terms of the Creative Commons Attribution License (CC BY). The use, distribution or reproduction in other forums is permitted, provided the original author(s) or licensor are credited and that the original publication in this journal is cited, in accordance with accepted academic practice. No use, distribution or reproduction is permitted which does not comply with these terms. 